\title{
Capture approximations beyond a statistical quantum mechanical method for atom-diatom reactions
}

\author{
Lizandra Barrios, Jesús Rubayo-Soneira \\ Instituto Superior de Tecnologías y Ciencias Aplicadas, \\ Ave. Salvador Allende y Luaces Quinta de los Molinos, Plaza, La Habana 10600, Cuba \\ Tomás González-Lezana* \\ Instituto de Física Fundamental (CSIC), \\ IFF-CSIC, Serrano 123, 28006 Madrid, Spain
}

(Dated: January 22, 2016; to submit to European Journal of Physics D)

\begin{abstract}
Statistical techniques constitute useful approaches to investigate atom-diatom reactions mediated by insertion dynamics which involves complex-forming mechanisms. Different capture schemes based on energy considerations regarding the specific diatom rovibrational states are suggested to evaluate the corresponding probabilities of formation of such collision species between reactants and products in an attempt to test reliable alternatives for computationally demanding processes. These approximations are tested in combination with a statistical quantum mechanical method for the $\mathrm{S}+\mathrm{H}_{2}(v=0, j=1) \rightarrow \mathrm{SH}+\mathrm{H}$ and $\mathrm{Si}+\mathrm{O}_{2}(v=0, j=1) \rightarrow \mathrm{SiO}+\mathrm{O}$ reactions, where this dynamical mechanism plays a significant role, in order to probe their validity.

PACS numbers:
\end{abstract}

\footnotetext{
* Email: t.gonzalez.lezana@csic.es
} 


\section{INTRODUCTION}

Since the pioneering work by Light [1-6] and Miller [7], statistical techniques have become useful methods to investigate the dynamics of atom-diatom reactive collisions mediated by the formation of an intermediate complex between reactants and products. The main assumption in these approaches relies on the possibility to treat the whole process separated in two stages: first the formation of the complex after the collision between the initial atom and the diatomic molecule and second, the fragmentation of the collision species to yield the products of the reaction. One of the key issues is then to calculate the probabilities for the complex to be formed just before reaching the intermediate region for different initial rovibrational states of the diatomic reactant. Different criteria, usually taken into account in order to estimate those capture probabilities simply as the unity or zero, depend on whether or not (i) the orbital angular momentum takes allowed values [8]; (ii) the impact parameter exceeds certain maximum value $[5,7,9,10]$ or (iii) the corresponding molecular state was energetically open. More elaborate approaches employ the microcanonical flux through the surface dividing the configuration space between reactants, the intermediate complex and products $[11,12]$. More recently a stastistical quantum mechanical (SQM) model was developed $[13,14]$ in which the capture probabilities were calculated in the most accurate way employing exact quantum approaches on ab initio potential energy surfaces (PESs). The method, succesfully employed in a large range of different atom-diatom reactions [15], implies the time-independent propagation of the wave function but versions which considered wave packet propagations in the time domain [16-19] and even quasi-classical trajectories (QCT) simulations [20-22] were soon reported.

Despite the noticeable reduction of computational effort gained with the neglection of the intermediate region in the calculations, there are situations which comprise considerable numerical difficulties. Thus, diatoms supporting a large number of rovibrational can still make SQM reactive scattering calculations expensive. In some cases, such as the $\mathrm{O}\left({ }^{1} D\right)+\mathrm{HCl}$ reaction [23], approximations limiting the number of rotational states in one of the product arrangements $(\mathrm{ClO}+\mathrm{H})$ have been employed. Due to the large number of $\mathrm{ClO}\left(v^{\prime \prime}, j^{\prime \prime}\right)$ states, the SQM calculations were performed over different $\left[j_{a}^{\prime \prime}, j_{b}^{\prime \prime}\right]$ subsets and the convergence of the corresponding capture probabilities was tested by variations of the actual dimensions of such interval. For more complex reactions involving four atoms, such as $\mathrm{CH}+\mathrm{H}_{2} \rightarrow \mathrm{CH}_{3}+\mathrm{H}$, 
a procedure combining the calculation of capture probabilities by means of time dependent approaches and estimates based on the number of available channels was attempted [24].

The main goal of this work is to test different possibilities to estimate the capture probabilities based on assumptions regarding energy values of the diatomic states and their rotational quantum number. The comparison with the actual values obtained by means of the above mentioned SQM method will enable us to validate the suggested approaches and to analyze the intrinsic perfomance of statistical techniques regarding these specific aspects. One of the examples chosen for this test is the $\mathrm{S}\left({ }^{1} D\right)+\mathrm{H}_{2} \rightarrow \mathrm{SH}+\mathrm{H}$ process, considered as an exothermic prototipical insertion reaction with a potential well about 4.23 eV depth between reactants and products. The collision has been studied in different occasions by means of statistical methods $[14-16,22,25,26]$, and the comparison with both exact quantum mechanical (QM) and experimental results provide solid evidences of the complex-forming character of the process on the ground electronic state. In particular, both differential cross sections (DCSs) and product translational energy (PTE) distributions of $\mathrm{S}+\mathrm{H}_{2}$ at a collision energy, $E_{c}$, of $97 \mathrm{meV}$ calculated by means of a exact time independent QM approach [27] and measured experimentally [28] were fairly well reproduced with the SQM method [15]. The agreement was extended to theoretical rotational distributions [27] and to the experimental DCS and PTE for the $\mathrm{S}+\mathrm{D}_{2}$ isotopic variant at $E_{c}=230 \mathrm{meV}$ [29].

Whereas exact QM calculations are feasible for $\mathrm{S}+\mathrm{H}_{2}$, the theoretical investigation of $\mathrm{Si}+\mathrm{O}_{2}(v=0, j=1) \rightarrow \mathrm{SiO}+\mathrm{O}$ is hindered by the existence of a multiwell structure in the PES which characterizes the reaction and a large number of $\mathrm{O}_{2}\left(v^{\prime}, j^{\prime}\right)$ rovibrational states $[30,31]$. The process is exothermic with a difference of about $0.93 \mathrm{eV}$ between the $\mathrm{Si}+\mathrm{O}_{2}$ reactants and the $\mathrm{SiO}+\mathrm{O}$ product arrangement and the overall dynamics is strongly mediated by minima of $2.65 \mathrm{eV}$ for the $\mathrm{Si}-\mathrm{O}-\mathrm{O}$ structure, $4.78 \mathrm{eV}$ for a $\widehat{\mathrm{OSiO}}$ geometry and of about $7.11 \mathrm{eV}$ for the $\mathrm{O}-\mathrm{Si}-\mathrm{O}$ linear arrangement. In fact, all investigations on the reaction consist on QCT calculations and no QM studies have been reported to date. In addition, the SQM calculation performed in Ref. [32] was limited to the computationally cheaper centrifugal sudden (CS) approximation version in which couplings between different values of $\Omega$ the third component of the rotational angular momemtum is neglected. The dynamics of the process is found to be the result of a competition between abstraction and insertion mechanisms. Thus, as either the collision energy or the initial rotational excitation of $\mathrm{O}_{2}$ is increased, the direct reaction pathway starts to shift towards a complex-forming 
mediated process [32]. A similar dynamical transition was also reported for the $\mathrm{H}+\mathrm{O}_{2}$ reaction $[33,34]$.

Results obtained by means a SQM and phase-space theory approaches are seen to agree with QCT calculations as the energy increases from $E_{c}=5 \mathrm{meV}$ up to $400 \mathrm{meV}$. The analysis of possible simplifying schemes to evaluate the capture probabilities for these kinds of systems without losing any of the overall features reproduced by the SQM approach becomes then a very interesting task.

This paper is organized as follows: First, general details of the SQM method and the procedures to estimate capture probabilities are given in Section II; Results are shown in Section III and discussed in Section IV. Conclusions are presented in Section V.

\section{THEORY}

According to the SQM approach of Ref. $[13,14]$ the total state-to-state reaction probability for an atom-diatom process for the total angular momentum $J$ and at an energy $E$, can be approximated as:

$$
\left|S_{v j \rightarrow v^{\prime} j^{\prime}}^{J}(E)\right|^{2} \approx \frac{p_{v j}^{J}(E) p_{v^{\prime} j^{\prime}}^{J}(E)}{\sum_{v^{\prime \prime} j^{\prime \prime}} p_{v^{\prime \prime} j^{\prime \prime}}^{J}(E)},
$$

where $p_{v j}^{J}$ refers to the capture probability to form the intermediate complex from the reactant diatom, $\mathrm{BC}$, in its $(v, j)$ rovibrational state and $p_{v^{\prime} j^{\prime}}^{J}$ the corresponding probability for the complex to fragmentate to the product diatom, AB, in its $\left(v^{\prime} j^{\prime}\right)$ rovibrational state. The sum in the denominator of Eq. (1) runs for all energetically open rovibrational states both for reactants and products.

These capture probabilities are obtained by solving the corresponding coupled channel equations resulting from the standard time-independent Schrödinger equation for the relative motion between the atom $\mathrm{A}$ and the diatomic molecule $\mathrm{BC}$ by means of a log derivative method. In particular, and after imposing the corresponding initial conditions for the coupling matrix at $R=R_{c}$, the capture radius which defines the region where the intermediate complex exists, the individual probabilities are obtained via the lack of unitarity of the corresponding scattering matrix: 


$$
p_{i}(E)=1-\sum_{i i^{\prime}}\left|S_{i i^{\prime}}(E)\right|^{2} .
$$

One of the main differences of this SQM method with respect to some other cruder statistical approaches is that the calculation of these capture probabilities is performed by means of accurate QM methods and using fully converged ab initio potential energy surfaces. In an attempt to reduce the computational cost it is possible to envisage further approximations to estimate the value of the individual capture probabilities following either energetic or kinematic considerations. Figure 1 depicts the energy diagram corresponding to potential curves of a general process for a specific rovibrational state of the product diatom defined as:

$$
V_{v j \Omega}^{J}(R)=\bar{V}(R)+V_{\text {cent }}^{J \Omega}(R)+E_{v j}
$$

where first term corresponds to the total potential integrated in the angle as in Ref. [35] as follows:

$$
\bar{V}(R)=\frac{1}{2} \int_{0}^{\pi} d \theta \sin \theta V\left(R, r_{\mathrm{eq}}, \theta\right)
$$

where $r_{\mathrm{eq}}$ is the diatom equilibrium distance, $E_{v j}$ is its energy and

$$
V_{\text {cent }}^{J \Omega}(R)=\frac{\hbar^{2}\left[J(J+1)-2 \Omega^{2}\right]}{2 \mu R^{2}}
$$

refers to the centrifugal term, being $\mu$ the reduced mass of the $\mathrm{A}+\mathrm{BC}$ system. The figure also includes the total energy at which we are performing the calculation, $E$, the values of the some rovibrational states, and the capture radius $R_{c}$ considered in the statistical approach.

The different schemes assumed to calculate the values of $p_{v j}^{J}(E)$ in Eq. (1) for the product arrangement are the following:

- $\operatorname{St}(E)$ : The $(v j)$ channel is energetically open if $E>V_{v j \Omega}^{J}\left(R_{c}\right)$ and $p_{v j}^{J}(E)=1$; It is taken as 0 if the energy condition is not satisfied;

- $\operatorname{St}(\Omega)$ : Capture probabilities for rovibrational channels with $\Omega>0$ are neglected if the above energy condition was not fulfilled for the corresponding $\Omega=0$ case; 
- $\operatorname{St}(\Omega, \Delta)$ : For those states which satisfy the two above conditions, the capture probability is taken as 1 if $E-V_{v j \Omega}^{J}\left(R_{c}\right)>\Delta$ and 0.5 if $E-V_{v j \Omega}^{J}\left(R_{c}\right)<\Delta$, where $\Delta$ is certain cutoff range which in this case is assumed as $0.1 \mathrm{eV}$.

In this work capture probabilities for the reactants are calculated by means of the SQM approach for each collisional energy. In these calculations, the maximum value of the total angular momentum, $J_{\max }$, accessible at that $E_{c}$ is employed as a limiting parameter for the product arrangement, where the capture probabilities are obtained by means of the approximations discussed above.

Once the capture probabilities are obtained, the state-to-sate cross sections are calculated according to the standard formulation:

$$
\sigma_{v j ; v^{\prime} j^{\prime}}(E)=\frac{\pi}{(2 j+1) k_{v j}^{2}} \sum_{J}(2 J+1)\left|S_{v j ; v^{\prime} j^{\prime}}^{J}(E)\right|^{2} .
$$

The calculation of the DCSs however, requires an additional approximation, in particular, the random phase approximation which neglects the cross terms between different partial waves in the expression usually employed in QM approaches. Within the SQM context the DCSs are obtained as follows:

$$
\sigma_{v, j ; v^{\prime}, j^{\prime}}(\theta, E) \simeq \frac{1}{8 k_{v j}^{2}} \frac{1}{(2 j+1)} \sum_{J \Omega^{\prime} \Omega}(2 J+1)^{2}\left[d_{\Omega^{\prime}, \Omega}^{J}(\pi-\theta)\right]^{2}\left|S_{v, j, \Omega ; v^{\prime}, j^{\prime}, \Omega^{\prime}}^{J}(E)\right|^{2},
$$

where the $d_{\Omega^{\prime}, \Omega}^{J}(\theta)$ quantities correspond to reduced Wigner rotation matrices.

\section{RESULTS}

Capture calculations as discussed in the previous sections have been carried out for the product arrangements of two processes: $\mathrm{S}+\mathrm{H}_{2}$ and $\mathrm{Si}+\mathrm{O}_{2}$. The differences regarding the complexity of the calculations for both processes are remarkable thus representing interesting benchmarks for the validity of the assumptions behind the above proposed approximations. The total reaction probability of Eq. (1) is obtained with individual probabilities for reactants $p_{v j}^{J}(E)$ calculated with the SQM approach and product probabilities $p_{v^{\prime} j^{\prime}}^{J}(E)$ estimated with the $\operatorname{St}(E), \operatorname{St}(\Omega)$ and $\operatorname{St}(\Omega, \Delta)$ approaches. 


\section{A. The $\mathbf{S}+\mathbf{H}_{2}$ reaction}

We have started with the calculation of the reaction probabilities as a function of the total angular momentum $J$ at $E_{c}=97 \mathrm{meV}$. Results obtained with the SQM method for the total probability for $\mathrm{S}+\mathrm{H}_{2}(v=0, j=1)$ are in almost a perfect agreement with estimates from the other approaches as shown in Figure 2. The main differences are manifested when the final-state-selected process $\mathrm{S}+\mathrm{H}_{2}(v=0, j=1) \rightarrow \mathrm{SH}\left(v^{\prime}\right)+\mathrm{H}$ is investigated. None of the capture schemes under consideration seem to reproduce correctly the formation of the SH diatom in its first vibrational excited state, with null probabilities for partial waves beyond $J_{\max }=14$. Up to $J \approx 5$, the comparison with the SQM results for the production of $\mathrm{SH}\left(v^{\prime}\right)$ both on its ground state and vibrationally excited reveals that the $\operatorname{St}(E)$ and $\operatorname{St}(\Omega, \Delta)$ approaches are in perfect accord with the QM method, but beyond that value of the total angular momentum, the probability for $\mathrm{SH}\left(v^{\prime}=1\right)$ decays much more rapidly. The SQM result for that channel, on the contrary, extends up to $J_{\max }=25$. The overestimation of the $\operatorname{SH}\left(v^{\prime}=0\right)$ case by the $\operatorname{St}(E)$ and $\operatorname{St}(\Omega, \Delta)$ results for $J \gtrsim 5$ compensates the differences to yield an overall agreement with the total probability. Interestingly, the stateto-state probabilities calculated by means of the $\operatorname{St}(\Omega)$ procedure are the results exhibiting the largest differences with respect to the SQM values, overestimating the formation of vibrationless $\mathrm{SH}$ but underestimating the prediction for the vibrationally excited product diatom. As for the range of partial waves in which the other suggested approximations also differ from the SQM values, the $P_{v^{\prime}}^{J}$ estimates compensate to yield total probabilities in good accord with such a method.

A much finer detail can be obtained via the rovibrational cross sections shown in Figure 3. None of the methods provide a perfect qualitative description of the SQM distributions, which display a maximum at a higher rotational state, $j^{\prime}=14$, than the rest: 9 , the $\operatorname{St}(\Omega \Delta)$ calculation, and 10 the two other approaches $\operatorname{St}(E)$ and $\operatorname{St}(\Omega)$. Moreover, all cross sections extend to larger $\operatorname{SH}\left(v^{\prime}=0, j^{\prime}\right)$ states, and in fact, the sudden cutoff seen for both the $\operatorname{St}(\Omega)$ and $\operatorname{St}(\Omega, \Delta)$ approaches invites to think on a too crude limitation of contributing rovibrational states with a non null capture probabilities. The purely energetic criterion assumed within the $\operatorname{St}(E)$ framework produces on the contrary population on too high rotational states.

Some of the behaviour observed for the reaction probabilities is recovered for the ICSs 
and thus the agreement between the SQM and the other statistical approaches for $\sigma_{\text {tot }}$ seems to be the result of an almost perfect compensation between the differences observed for the state-to-state probabilities. Table I shows that the predictions for the total ICS at $E_{c}=97$ $\mathrm{meV}$ are almost identical in all cases but, on the contrary, the production of $\mathrm{SH}$ in its vibrational ground state seems to be overestimated with the statistical approximations with respect to the $\mathrm{SQM}$ result for $\sigma_{v^{\prime}=0}$ whereas noticeably less $\mathrm{SH}\left(v^{\prime}=1\right)$ is predicted with these methods.

The interesting result regards then more averaged quantities with no specified rovibrational final state of the SH product diatom. Figure 4 shows the corresponding DCSs for the $\mathrm{S}+\mathrm{H}_{2}(v=0, j=1) \rightarrow \mathrm{SH}+\mathrm{H}$ reaction at the collision energy here under study. The SQM angular distribution, which conveniently averaged with the result for the reaction initiated from $\operatorname{SH}(v=0, j=0)$ in its ground state, was in such a good agreement with both exact QM and experimental cross sections [15], is well reproduced by all statistical approximations. Despite slight deviations at specific values of the angle, the results shown in Figure 4 certainly validate the approximations as even cheaper counterparts of the SQM approach.

\section{B. The $\mathrm{Si}+\mathrm{O}_{2}$ reaction}

Besides the test shown in the previous subsection for the $\mathrm{S}+\mathrm{H}_{2}$ reaction, it is interesting to analyze the feasibility of the proposed approximative capture schemes for computationally more demanding processes, such as $\mathrm{Si}+\mathrm{O}_{2}$. We start our study of this collision initiated with molecular oxygen on its ground rovibrational state $\mathrm{O}_{2}(v=0, j=1)$ at a similar energy as in the above discussion. Figure 6 shows the corresponding reaction probabilities as a function of the total angular momentum, $P(J)$, at $E_{c}=100 \mathrm{meV}$ obtained by means of the statistical treatments under consideration in this work. The agreement for the total probability among all theoretical approaches is remarkable but the results shown for specific final vibrational states of the $\mathrm{SiO}\left(v^{\prime}\right)$ product diatom (in particular $v^{\prime}=1$ and 5) reveal that some sort of compensation is also taking place for the $\operatorname{St}(\Omega, \Delta)$ calculation. More precisely, the probability predicted by this procedure for the case of formation of $\operatorname{SiO}\left(v^{\prime}=1\right)$ is noticeable larger at some partial waves in comparison with the SQM method, whereas is slightly smaller for $v^{\prime}=5$. The interesting result however is that, as opposed to the case of $\mathrm{S}+\mathrm{H}_{2}$, the rest of capture approximations seem to start in good accord with the SQM 
predictions.

The analysis at the rotational state level is performed via the corresponding state-to-state cross sections for the $\mathrm{Si}+\mathrm{O}_{2}(v=0, j=1) \rightarrow \mathrm{SiO}\left(v^{\prime}, j^{\prime}\right)+\mathrm{O}$ reaction. The corresponding comparison with the SQM distributions shown in Figure 6 for the specific case $v^{\prime}=0$ reveals the trend of the approximative capture schemes to produce rotational ICSs extending to larger excited $j^{\prime}$ levels. $\operatorname{St}(E)$ and $\operatorname{St}(\Omega)$ are found to yield ditributions with the maximum located at slightly higher rotational states, and are almost identical apart of the sudden cutoff displayed by the latter around $j^{\prime} \sim 110$. The same forced trend at the tail of the distributions is observed for the $\operatorname{St}(\Omega, \Delta)$ calculation. The remarkable point is that populations for lower rotational states, $j^{\prime} \lesssim 90$, predicted with this approach are in an overall good agreement with the SQM cross sections.

This general good result obtained for the rotational cross sections extends to the vibrational distributions. In Figure 7 we show the corresponding cross sections for the $\mathrm{Si}+\mathrm{O}_{2}(v=0, j=1) \rightarrow \mathrm{SiO}\left(v^{\prime}\right)+\mathrm{O}$ at three different collision energies: $5 \mathrm{meV}$ (top panel); $100 \mathrm{meV}$ (middle panel) and $400 \mathrm{meV}$ (bottom panel). The sum on all rotational states of distributions shown in Figure 6 leads to the first point of the middle panel for vibrational cross sections for $v^{\prime}=0$ at $100 \mathrm{meV}$. The agreement observed for this specific case certainly comprises the balance between slight discrepancies regarding the populations according to the different approximations with respect to the SQM predictions, but the comparison is much more favourable that the $\mathrm{S}+\mathrm{H}_{2}$ case. The good performance of the $\operatorname{St}(E)$, St $(\Omega)$ and $\operatorname{St}(\Omega, \Delta)$ schemes extends both to other vibrational states of $\operatorname{SiO}\left(v^{\prime}\right)$ for the same collision energy and to other collision energies. Specially good in the case of $E_{c}=5 \mathrm{meV}$, slight deviations from the SQM cross sections are noticed for some vibrational distributions obtained at $400 \mathrm{meV}$. In particular, the lowest $\mathrm{SiO}\left(v^{\prime}\right)$ states are less populated in the $\mathrm{St}(E)$ calculation than in the rest.

The total DCSs for the $\mathrm{Si}+\mathrm{O}_{2}$ reaction at $E_{c}=100 \mathrm{meV}$ calculated with the statistical approaches considered in this work are compared in Figure 8. The agreement near the sideways scattering direction $\theta \sim 90^{\circ}$ is noticeable, but outside the angular range between $\sim 45^{\circ}$ and $\sim 135^{\circ}$ some deviations in comparison with the SQM distribution are manifested. The rest of capture approaches yield DCSs which are slightly below the SQM result, about 1.1 times larger at the strict peaks along $0^{\circ}$ and $180^{\circ}$. 


\section{DISCUSSIONS}

Given that the proposed approximations are only applied to the calculation of capture probabilities for the products arrangement, it is an expected result that the observed differences with respect to the SQM method are mainly observed when the final rovibrational state is specified. More averaged quantities however have been found in a reasonably good agreement which in general prove the validity of the tested approaches. The selection of the right parameter to use as criterion in the approximations constitutes one of the crucial issues in this kind of study. In view of the present results one would say that purely energetic considerations, as considered in the $\operatorname{St}(E)$ option, are not sufficient as they yield the most significant deviations in rotational distributions (see Figs. 3 and 6) and the highest collisional energy considered for the $\mathrm{Si}+\mathrm{O}_{2}$ case (see bottom panel of Fig. 7). This seems to be consistent with the procedures followed for instance in the phase space theory by Larrégaray et al. [25] where limitations on the total angular momentum are imposed in order to assign the unity to the corresponding capture probability. Analogously, Miller considered the impact parameter as the determining factor to discriminate between 0 and 1 for these probabilities [7]. The procedures which add an extra requirement on the final rotational states, that is $\operatorname{St}(\Omega)$ and $\operatorname{St}(\Omega, \Delta)$, produce distributions with usually too abrupt endings. In this sense, the examples under consideration here, suggest that the result can differ depending on the number of final states sharing the capture probabilities.

One of the key issues of these types of approximations regards the computational time saved with respect to a more complete calculation. In this sense, the performance of the proposed approaches is clearly unbeatable. One of the hardest cases shown in this paper, the $\mathrm{Si}+\mathrm{O}_{2}$ reaction at $E_{c}=400 \mathrm{meV}$, can be solved in less than one hour whereas the corresponding SQM calculations can take an entire week. The difference becomes even more remarkable when the system under study gets more demanding in terms of numerical effort. Preliminary investigations on reactions such as $\mathrm{Si}+\mathrm{OH}$ reveals that several months calculations can be approximated by means of these cheaper methods with runs of less than a couple of hours of computing time.

Improvements and refinements are clearly possible. Thus, for example, it might be argued that the calculation of the average potential in Eq. (4) could be carried out by the explicit solution of the involved integrals instead of choosing the equilibrium distance of the product 
diatom. That would indeed provide a better average description of the potential energy landscape for the reaction but in turn, would cause a significant increase of the computational difficulty and therefore, out of the scope of the simplicity as a main goal of this work. Similar considerations can be taken into account when the proposed methods employs merely the value of the entire potential written in Eq. (3) at the capture radius, $R_{c}$. This choice, which in some way, supports the choice of such a parameter within the context of the SQM approach defining the region at which the intermediate complex is assumed to form, can lead to a not necessarily good value of the potential when one is trying to know if a specific rovibrational state is energetically open or not. Potential curves displayed in Figure 1 show how different the conclusion can be depending on the precise value of $R$. In this sense, the actual calculation performed in the SQM method provides a much better picture with the complete $V(R)$ potential involved in the log-derivative propagation. In addition to this, and given the different contributions introduced by the centrifugal terms with values of $J$ and $\Omega$ the definition itself of an unique $R_{c}$ seems too restrictive. It would be desirable to develop a procedure to choose the capture radius for each set of $(J, \Omega)$ values, depending, for instance, on the presence of centrifugal barriers.

One of the most interesting applications of these approaches could be the framework of more complicated systems such as four atoms collisions [24]. $\mathrm{AB}+\mathrm{CD}$ or $\mathrm{A}+\mathrm{BCD}$ reactions constitute an ideal scenario to employ approximations which may help to simplify the calculations. Procedures to assign capture probabilities to asymptotic bound states of either reactants or products based on energetic and kinematic considerations in terms of the energy of the specific states can become an useful alternative to sometimes prohibitive exact QM calculations.

\section{CONCLUSIONS}

Different approaches to estimate the capture probabilities within the context of statistical techniques have been tested with two atom-diatom reactions: $\mathrm{S}+\mathrm{H}_{2}(v=0, j=1) \rightarrow \mathrm{SH}+\mathrm{H}$ and $\mathrm{Si}+\mathrm{O}_{2}(v=0, j=1) \rightarrow \mathrm{SiO}+\mathrm{O}$. Reaction probabilities, rovibrational distributions, integral and differential cross sections have been calculated by means of these approximations and compared with results obtained with a statistical quantum mechanical method previously employed for these same processes. Total magnitudes are in general well described 
with the proposed methods and only at the state-to-state level some differences are manifested with respect to the quantum mechanical method, with compensations between the predictions for the different final vibrational state of the product diatoms. Rotational cross sections are perhaps the most sensitive magnitudes to the precise approximation assumed, with relevant deviations when only energy considerations on the diatom rovibrational states $\left(v^{\prime}, j^{\prime}\right)$ are taken into account to assign non-null probabilities. In turn, distributions with possibly too abruptly ended tails are obtained when extra considerations regarding rotational quantum numbers and their third components are included in the estimation of the capture probabilities. The interesting thing is that the comparison between the performance of the different schemes in the two processes under consideration suggests that in fact the approximations work better for those cases mediated by a large number of rovibrational states. It is precisely for those computationally more demanding reactions in which these kinds of simplistic capture techniques can be more useful.

\section{ACKNOWLEDGMENTS}

TGL would like to thank support from MICINN with Grants No. FIS2011-29596-C02-01 and FIS2014-51993-P, and LB acknowledges support from CSIC i-COOP+ Program Ref. COOPA20039.

All authors have contributed equally to the paper. 


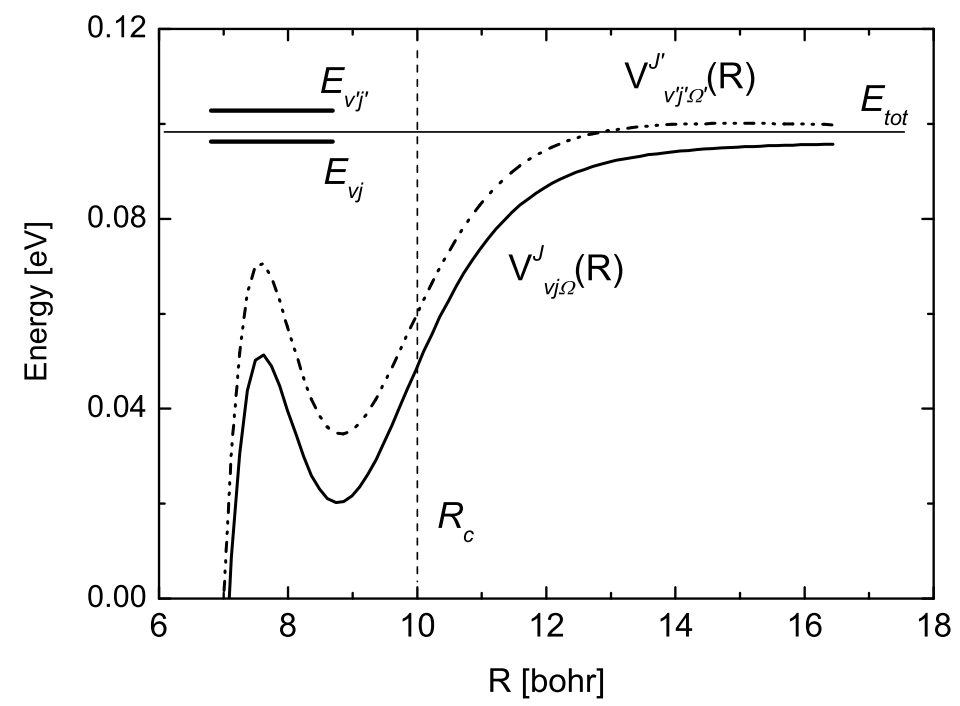

FIG. 1: Scheme with the potential energy curves for a general process corresponding to specific values of the total angular momentum, $J$, the diatom vibrational state, $v$, the rotational quantum number, $j$, and its third component, $\Omega$. Values of the total energy and the corresponding rovibrational levels of the diatom are also indicated. $R_{c}$ refers to the capture radius in the SQM approach. 


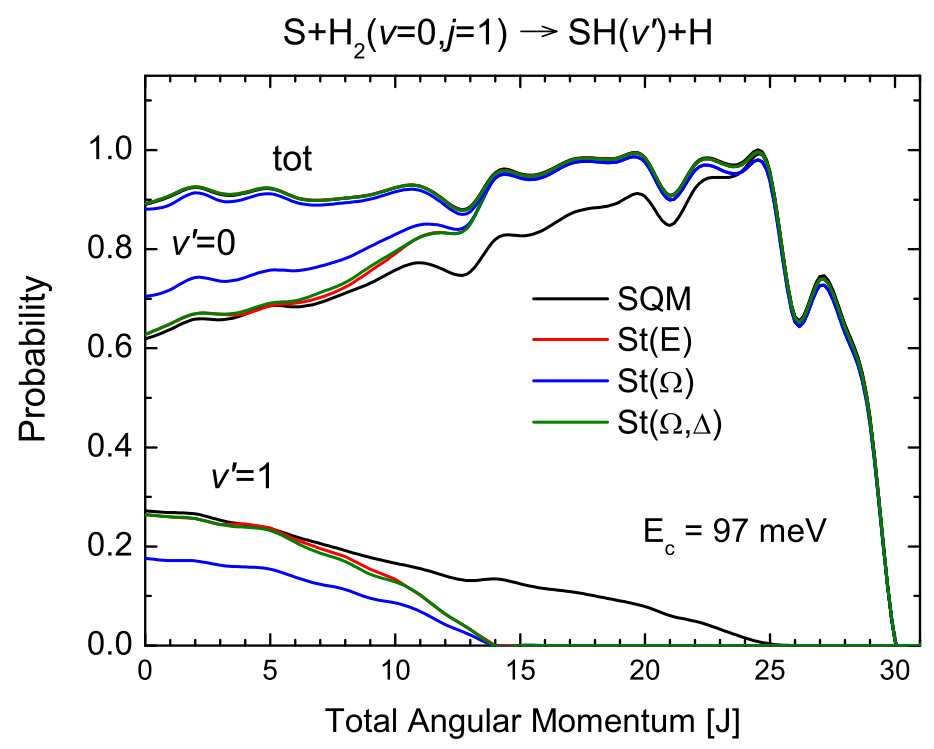

FIG. 2: Total and final-vibrational-state resolved reaction probability as a function of $J$ for the $\mathrm{S}+\mathrm{H}_{2}(v=0, j=1) \rightarrow \mathrm{SH}\left(v^{\prime}=0,1\right)+\mathrm{H}$ reaction at $97 \mathrm{meV}$ collision energy obtained by means of the SQM method (black lines) and the different statistiscal approaches proposed in this paper: $\operatorname{St}(E)$ (red lines), $\operatorname{St}(\Omega)$ (blue lines) and $\operatorname{St}(\Omega, \Delta)$ (green lines). 


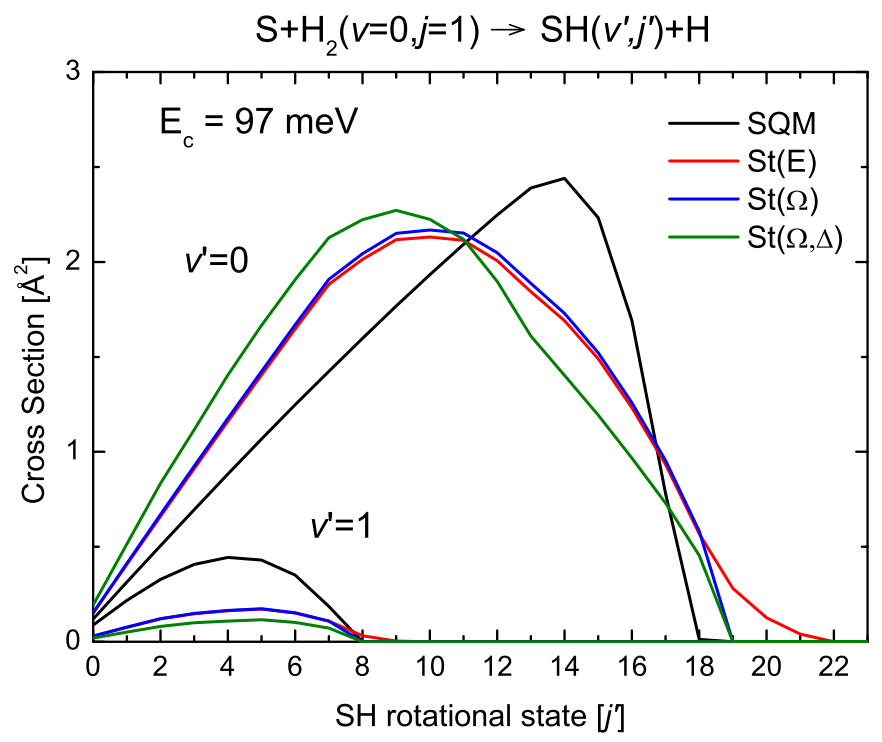

FIG. 3: Rotational distributions for the $\mathrm{S}+\mathrm{H}_{2}(v=0, j=1) \rightarrow \mathrm{SH}\left(v^{\prime}=0,1\right)+\mathrm{H}$ reaction at $E_{c}=$ $97 \mathrm{meV}$, calculated with the statistical methods here discussed. Colors are as in Figure 2. Units are $\AA^{2}$. 


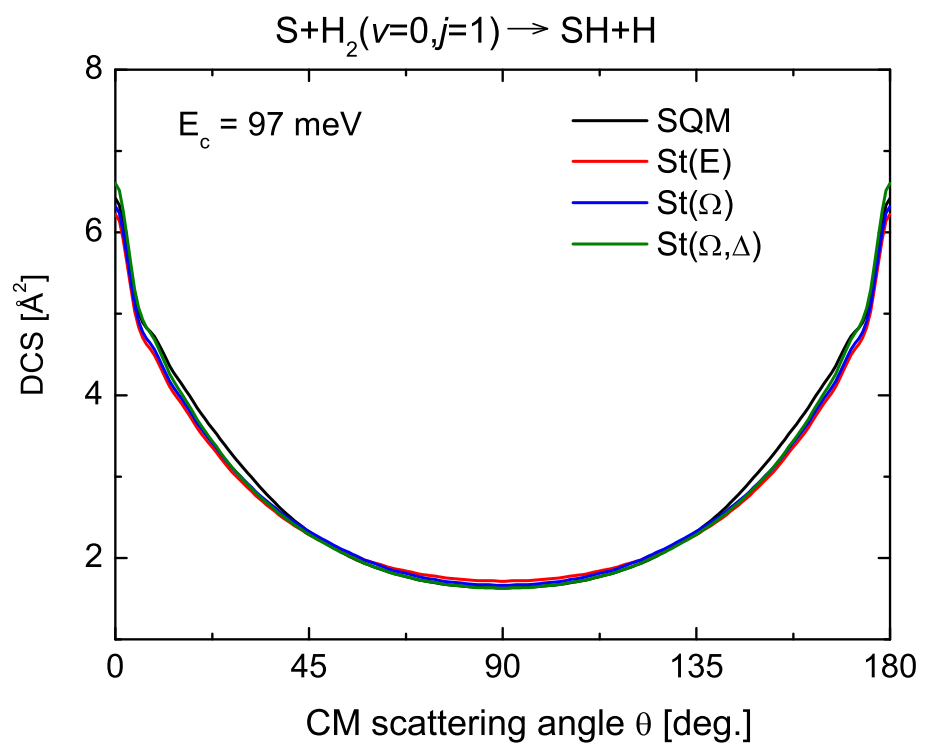

FIG. 4: Total differential cross sections in $\AA^{2}$ for $\mathrm{S}+\mathrm{H}_{2}(v=0, j=1) \rightarrow \mathrm{SH}+\mathrm{H}$ at $E_{c}=97 \mathrm{meV}$. Colors are as in Figure 2. 


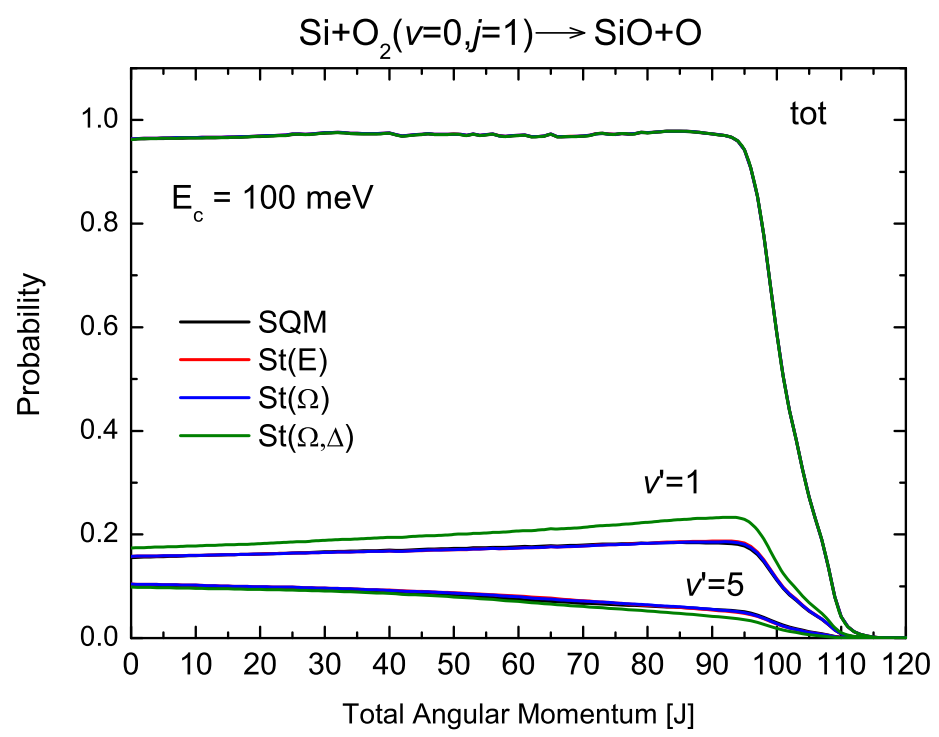

FIG. 5: Total and final-vibrational-state-selected probability as a function of $J$ for the $\mathrm{Si}+\mathrm{O}_{2} \rightarrow$ $\mathrm{SiO}\left(v^{\prime}=1,5\right)+\mathrm{O}$ reaction at $E_{c}=100 \mathrm{meV}$. Colors correspond to the different statistical methods employed as in Figure 2. 


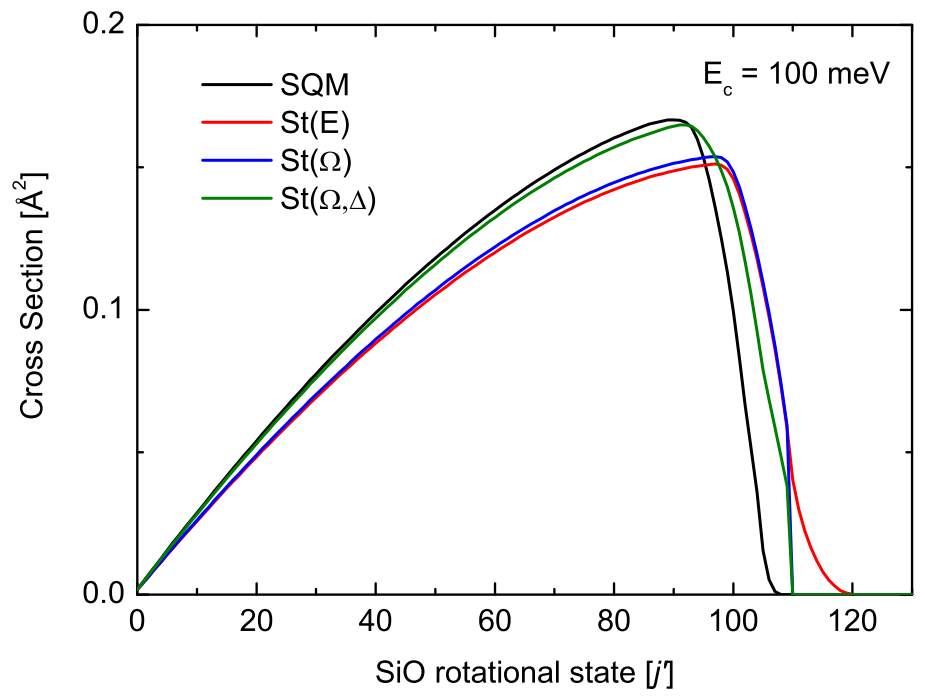

FIG. 6: Rotational distributions for the $\mathrm{Si}+\mathrm{O}_{2} \rightarrow \mathrm{SiO}\left(v^{\prime}=0, j^{\prime}\right)+\mathrm{O}$ reaction at $E_{c}=100 \mathrm{meV}$. Colors are as in Fig. 3 and units are $\AA^{2}$. 


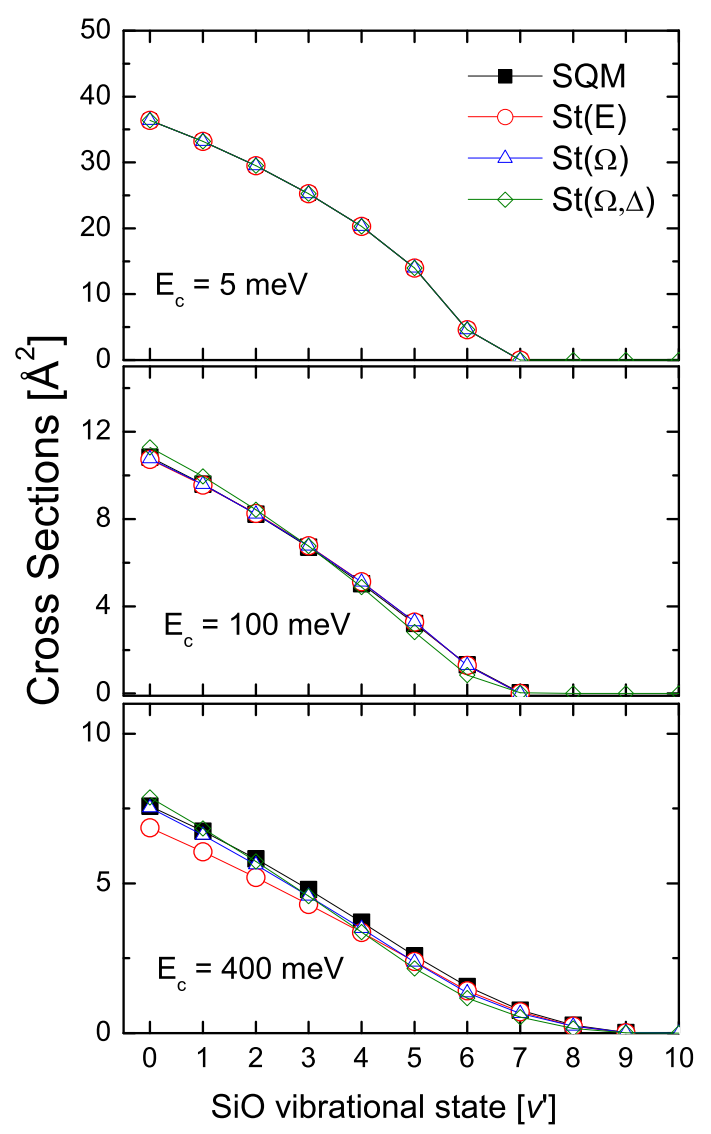

FIG. 7: Vibrational distributions for the $\mathrm{Si}+\mathrm{O}_{2} \rightarrow \mathrm{SiO}\left(v^{\prime}\right)+\mathrm{O}$ reaction at different collisional energies: $5 \mathrm{meV}$ (top panel); $100 \mathrm{meV}$ (middle panel) and $400 \mathrm{meV}$ (bottom panel). Units are $\AA^{2}$ and black lines and full squares are for the SQM approach; red lines and empty circles ate cross sections obtained with the $\operatorname{St}(E)$ version; blue lines and empty triangles with the $\operatorname{St}(\Omega)$ approximation and green lines and empty diamonds are for results with the $\operatorname{St}(\Omega, \Delta)$ method. 


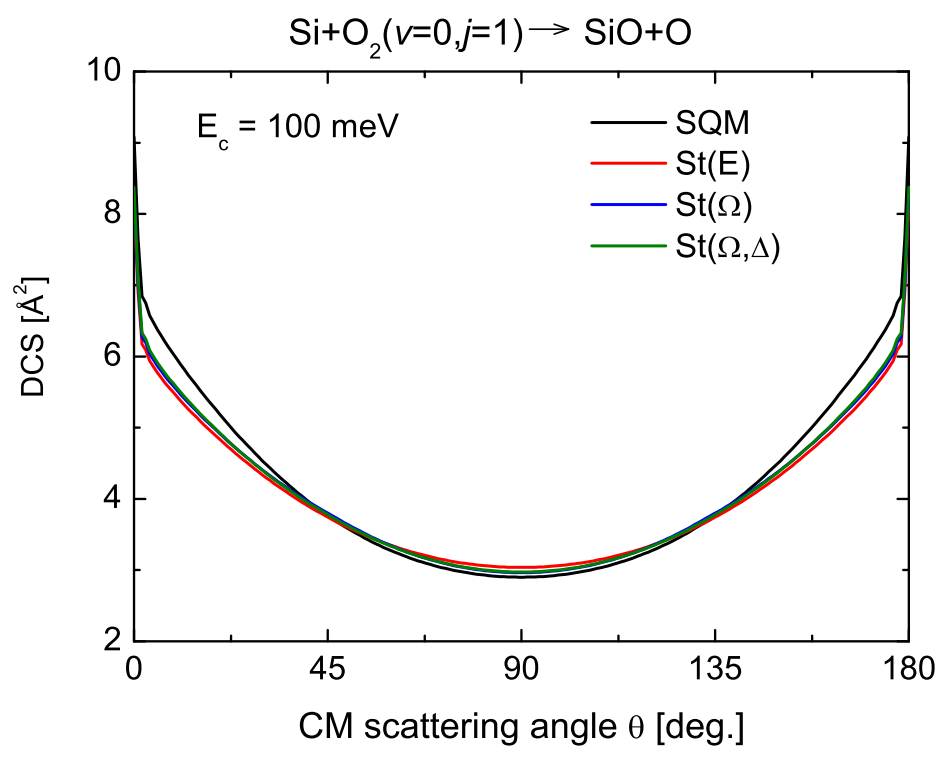

FIG. 8: Total DCS for the for $\mathrm{Si}+\mathrm{O}_{2}(v=0, j=1) \rightarrow \mathrm{SiO}+\mathrm{O}$ at $E_{c}=100 \mathrm{meV}$. Colors are as in Figure 4. 
TABLE I: Total and final-vibrational-state-selected ICSs for $\mathrm{S}+\mathrm{H}_{2}(v=0, j=1) \rightarrow \mathrm{SH}\left(v^{\prime}\right)$ at $E_{c}=97 \mathrm{meV}$ obtained by means of the different statistical approaches. Units are $\AA^{2}$.

\begin{tabular}{|c|ccc|}
\hline \hline & $\sigma_{\text {tot }}$ & $\sigma_{v^{\prime}=0}$ & $\sigma_{v^{\prime}=1}$ \\
\hline $\mathrm{SQM}$ & 27.89 & 25.44 & 2.45 \\
$\mathrm{St}(E)$ & 27.82 & 26.82 & 1.00 \\
$\mathrm{St}(\Omega)$ & 27.82 & 26.84 & 0.98 \\
$\mathrm{St}(\Omega, \Delta)$ & 27.51 & 26.86 & 0.65 \\
\hline \hline
\end{tabular}

[1] J. C. Light, J. Chem. Phys. 40, 3221 (1964).

[2] P. Pechukas and J. C. Light, J. Chem. Phys. 42, 3281 (1965).

[3] J. C. Light and J. Lin, J. Chem. Phys. 43, 3209 (1965).

[4] J. Lin and J. Light, J. Chem. Phys. 45, 2545 (1966).

[5] P. Pechukas, J. C. Light, and C. Rankin, J. Chem. Phys. 44, 794 (1966).

[6] R. A. White and J. C. Light, J. Chem. Phys. 55, 379 (1971).

[7] W. H. Miller, J. Chem. Phys. 52, 543 (1970).

[8] J. C. Light, Discuss. Faraday Soc. 44, 14 (1967).

[9] W. B. Miller, S. A. Safron, and D. R. Herschbach, Discuss. Faraday Soc. 44, 108 (1967).

[10] S. Safron, N. Weinstein, D. Herschbach, and J. Tully, Chem. Phys. Lett. 12, 564 (1972).

[11] W. H. Miller, J. Chem. Phys. 65, 2216 (1976).

[12] E. Pollak and P. Pechukas, J. Chem. Phys. 70, 325 (1979).

[13] E. J. Rackham, F. Huarte-Larrañaga, and D. E. Manolopoulos, Chem. Phys. Lett. 343, 356 (2001).

[14] E. J. Rackham, T. González-Lezana, and D. E. Manolopoulos, J. Chem. Phys. 119, 12895 (2003).

[15] T. González-Lezana, Int. Rev. Phys. Chem. 26, 29 (2007).

[16] S. Y. Lin and H. Guo, J. Chem. Phys. 120, 9907 (2004). 
[17] S. Y. Lin and H. Guo, J. Chem. Phys. 122, 074304 (2005).

[18] S. Y. Lin and H. Guo, J. Phys. Chem. A 108, 10066 (2004).

[19] S. Y. Lin, E. J. Rackham, and H. Guo, J. Phys. Chem. A 110, 1534 (2006).

[20] F. J. Aoiz, V. Sáez Rábanos, T. González-Lezana, and D. E. Manolopoulos, J. Chem. Phys. 126, 161101 (2007).

[21] F. J. Aoiz, T. González-Lezana, and V. Sáez Rábanos, J. Chem. Phys. 127, 174109 (2007).

[22] F. J. Aoiz, T. González-Lezana, and V. S. Rábanos, J. Chem. Phys. 129, 094305 (2008).

[23] P. Bargueño, P. G. Jambrina, J. M. Alvariño, M. L. Hernández, F. J. Aoiz, M. Menéndez, E. Verdasco, and T. González-Lezana, J. Phys. Chem. A 113, 14237 (2009).

[24] J. Mayneris, A. Saracibar, E. M. Goldfield, M. González, E. García, and S. K. Gray, J. Phys. Chem. A 110, 5542 (2006).

[25] P. Larrégaray, L. Bonnet, and J.-C. Rayez, J. Phys. Chem. A 110, 1552 (2006).

[26] P. Larrégaray and L. Bonnet, Comp. Theor. Chem. 990, 18 (2012).

[27] L. Bañares, F. J. Aoiz, P. Honvault, and J.-M. Launay, J. Phys. Chem. A 108, 1616 (2004).

[28] S.-H. Lee and K. Liu, Appl. Phys. B 71, 627 (2000).

[29] S.-H. Lee and K. Liu, J. Phys. Chem. A 102, 8637 (1998).

[30] F. Dayou and A. Spielfiedel, J. Chem. Phys. 119, 4237 (2003).

[31] F. Dayou, W.-Ü. L. Tchang-Brillet, and M. Monnerville, J. Chem. Phys. 123, 084306 (2005).

[32] F. Dayou, P. Larrégaray, L. Bonnet, J.-C. Rayez, P. N. Arenas, and T. González-Lezana, J. Chem. Phys. 128, 174307 (2008).

[33] P. Bargueño, T. González-Lezana, P. Larrégaray, L. Bonnet, and J.-C. Rayez, Phys. Chem. Chem. Phys. 9, 1127 (2007).

[34] P. Bargueño, T. González-Lezana, P. Larrégaray, L. Bonnet, J.-C. Rayez, M. Hankel, S.C. Smith, and A.J.H.M. Meijer, J. Chem. Phys. 128, 244308 (2008).

[35] P. Larrégaray, L. Bonnet, and J.-C. Rayez, J. Chem. Phys. 127, 084308 (2007). 